\title{
Autonomous Swarm of UAVs for Tracking of Flying Insects with Harmonic Radar
}

\author{
A. Lavrenko ${ }^{\dagger \ddagger}$, Z. Barry*, R. Norman*, C. Frazer*, Y. Ma*, G. Woodward* and S. Pawson ${ }^{\dagger \S}$ \\ ${ }^{\dagger}$ Scion, New Zealand Forest Research Institute, Christchurch, New Zealand \\ ${ }^{\ddagger}$ Radio Systems Group, University of Twente, Enschede, Netherlands (current address) \\ ${ }^{*}$ Wireless Research Centre, University of Canterbury, Christchurch, New Zealand \\ $\S$ School of Forestry, University of Canterbury, Christchurch, New Zealand (current address) \\ Email: graeme.woodward@ canterbury.ac.nz, a.lavrenko@utwente.nl
}

\begin{abstract}
In this paper, we present a design and first proof-ofconcept test results of a distributed radio-localization system for tracking of moving targets with a swarm of drones. In particular, we develop and test a software framework that uses bistatic ranges to follow a target in-flight. We conduct full-system flight test with up to two drones in the air, three simulated drones, and a laptop equipped with a GPS module as the target. The system is shown to dynamically track the target as it moved, with both real and simulated drones following it in a specified formation.

Index Terms-harmonic radar, UAV swarm, insect tracking
\end{abstract}

\section{INTRODUCTION}

Globally, insect populations are in decline [1]. With thousands of insect species having limited, if any, behavioral information, having a way to learn what they do in the environment is essential for informed biosecurity, conservation and pest management efforts. Due to stringent size and weight restrictions, conventional radio-telemetry methods developed to track larger wild-life, including larger insects such as locusts, are ill-suited for operation with small flying insects. A possible solution is offered by applying the harmonic radar (HR) principle [2], [3]. HR is a type of non-linear radar that uses passive transponder tags to create a harmonic response from a target that is otherwise unlikely to produce a distinguishable radar response on its own. Since regular objects do not usually produce harmonic returns when illuminated with an RF signal, harmonic operation allows significant reduction of linear clutter produced by the environment, while the passive nature of harmonic tags provides an opportunity for tag minimization [4]-[6]. HR systems typically either use received signal strength (RSS)-based direction finding principle in short-range operation or high-power ranging with direction finding for operation over larger distances. The downside of using harmonic tags is their low power conversion efficiency [6], [7]. As a result, RSS-based systems are typically handheld and must be manually operated in a close proximity to the target [3]. Ranging HR systems on the other hand tend to be high power stationary systems (e.g., up to $1 \mathrm{KWatt}$ for $500 \mathrm{~m}$ of detection range [5]) that are often built by re-purposing maritime radar equipment.

This work was partially supported by the SSIF via Scion Postdoctoral fellowship S07084 and the Royal Society of New Zealand Catalyst:Seeding grant CSG-FRI1802. The authors thank K. Barnsdale and S. Schofield from the University of Canterbury Drone Laboratory for their support in field tests.
Recently, it has been suggested to use unmanned aerial vehicles (UAVs) as harmonic radar carriers [8]-[10]. Placing HR on an UAV could help alleviate some of the limitations of conventional HR systems. First, it ensures proximity to the target that reduces transmit power requirements enabling the use of lower power short-range devices. Using an aerial platform with an overhead view of the target also improves chances of the line-of-sight permitting more reliable localization. Finally, being able to follow an insect along its flight path allows access to remote areas and complex terrains. However, traditional radar target positioning based on direction finding in combination with ranging poses several problems for insect localisation and tracking on UAVs. Using a narrowbeam antenna to track a moving target from a mobile platform is problematic if the target is moving quickly and/or erratically. Placing a mechanically rotating antenna on a UAV on the other hand, is impractical due to the size and weight restrictions. An alternative is to use a multistatic setup with several timesynchronized receivers that employ multilateration to estimate target location.

In this paper, we discuss system-level design of such a distributed multistatic radar system to be placed and operated on a swarm of UAVs. With substantial research effort dedicated to the development of compact radar modules suitable for operation on drones [7], [10]-[13], here we focus on the software aspects of the system design necessary to enable an autonomous swarm of UAVs to track a moving target using multilateration. Particularly, we detail the design and implementation of the communication, localization and UAV swarming (CLS) framework, as well as the flight control system that enables the CLS operation. We perform proof-ofconcept lab and field trials with an emulated ranging scenario that allows us to test system functionality in a controlled environment. Our test results demonstrate practical feasibility of the proposed configuration. Furthermore, although this work was primarily motivated by an HR for insect tracking with UAVs application, the designed software framework can be easily adapted to serve any distributed localization system, either mobile or stationary, subject to the availability of backhaul communication links between the sensing nodes. 


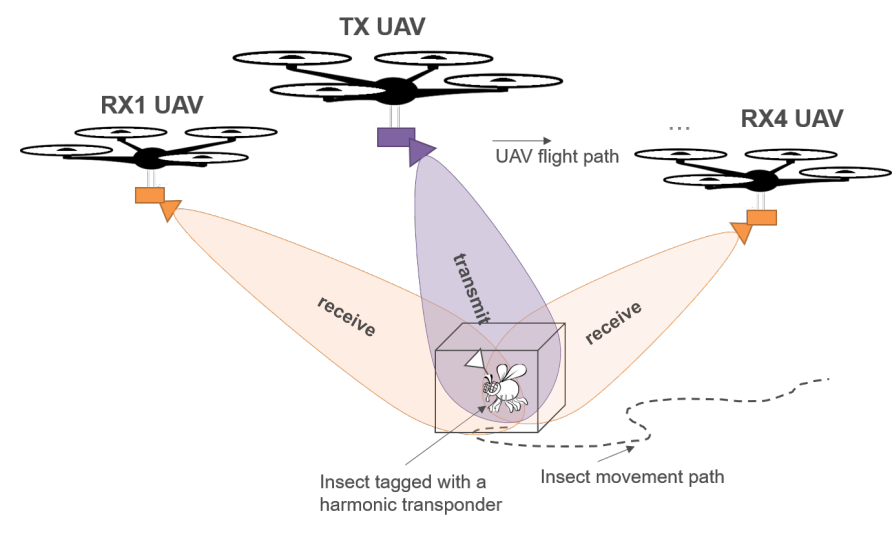

Fig. 1. Distributed multistatic HR system for tracking of flying insects from UAVs consisting of one TX UAV and up to 4 RX UAVs.

\section{SYSTEM OVERVIEW}

We consider a distributed multistatic configuration with one HR transmitter and four HR receivers that each are placed on a separate UAV, as illustrated in Fig. 1. The target location is estimated from the obtained bistatic ranges via multilateration. Radar units, both the transmitter and the receivers, are assumed to share a common time reference to accurately estimate the ranges to the target. Range measurements from each receiver UAV (RX UAV) together with their GPS coordinates are collected at a single node to derive the target location, which is then communicated back to the UAVs' flight control (FC). The UAV swarm then moves with the target in a pre-defined formation. From the system design viewpoint, the system from Fig. 1 comprises the following elements.

- Radar hardware: the radar sub-system includes the harmonic tag that must be placed on the insect as well as the radar hardware (receiver and transmitter units) that shall be located on the UAVs. For accurate range estimation from bistatic measurements, it also requires additional time-synchronisation between the transmitter and the receiver, e.g., provided by timing GPS modules.

- Communication: once individual range measurements are obtained, they must be sent together with the RX UAV locations to a centralised processing node for target localization. A natural choice for the central processing node is the transmitter UAV (TX UAV), as it is likely to be in the centre of the formation and will need to carry heavier equipment than the RX UAVs. Computed target location must then be sent back to the UAV FC systems, so that they can follow the target.

- Localisation: with one transmitter and four receivers the target location corresponds to the point of intersection of four spheroids with the foci determined by the UAV locations and equatorial lengths determined by the corresponding ranges. Finding the intersection point requires solving a system of non-linear equations in real time, i.e., at a given update rate.

- Swarming logic: the UAVs need to move in a coordinated formation, following the estimated target path, to avoid collisions with each other and the surroundings.

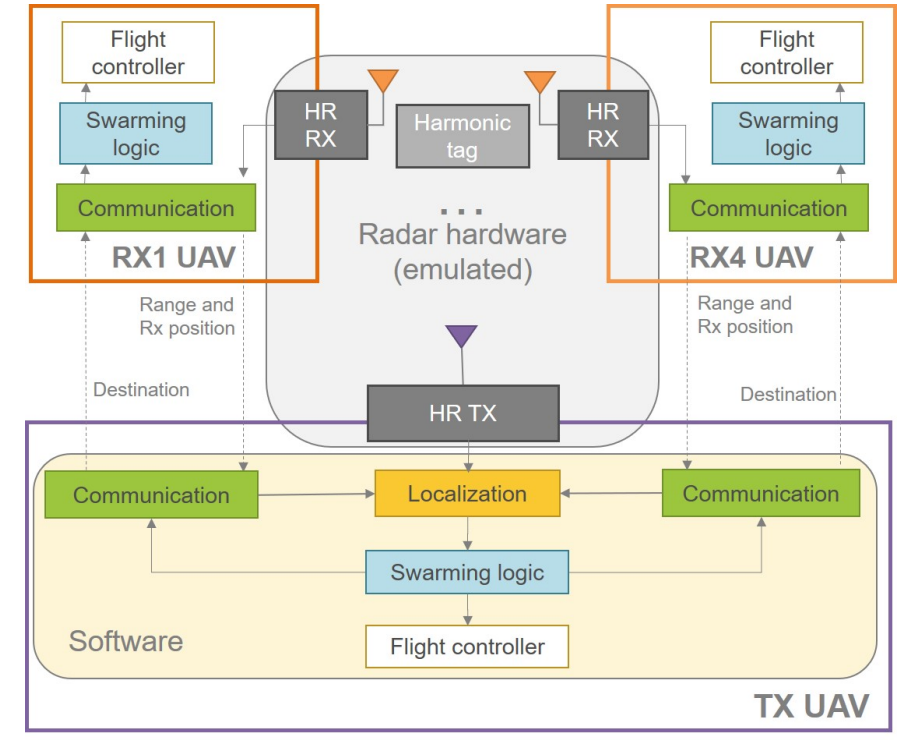

Fig. 2. Functional representation of an insect tracking system from Fig. 1.

The relation between the four sub-systems described above is illustrated in Fig. 2 in a simplified schematic form. It further distinguishes the "hardware" part that consists of the radar sub-system (HR transmitter, receivers and the harmonic tag) and the "software" framework that combines communication, localisation and UAV swarming (CLS). In the following, we detail the design of the latter, while various aspects of HR hardware design can be found in [4], [6], [7], [9]-[14].

\section{CLS FRAMEWORK}

The CLS framework is designed to accommodate five UAVs (one acting as a TX UAV and four as RX UAVs). The UAVs stay in a fixed formation with TX UAV in the centre and RX UAVs at fixed (equal) offsets from it. The TX UAV serves as the central processing and control node that computes target location based on the individual range readings from RX UAVs and ensures that UAVs stay in formation while in flight.

\section{A. Communication}

The aim of the communication sub-system is to implement bidirectional, reliable, low latency wireless communication between the TX UAV and four RX UAVs. The network also needs to be local, so that it could move together with the swarm. The UAVs used for system prototyping and testing were Aeronavics NAVI quadcopters. The NAVIs are equipped with an Intel NUC for on-board processing that has a builtin WiFi adapter. A natural solution was then to implement communication links over WiFi with one UAV, e.g., TX UAV, creating a WiFi hotspot which the others connect to. The NUCs also provide an IP/TCP stack, so that lower layer networking issues don't need to be considered.

There are two types of messages exchanged between the UAVs: each RX UAV sends an update to the TX for each new range reading, and the TX UAV sends an update to all RX UAVs for each new target position estimate. To implement the messaging, a ZeroMQ message passing library was used. The 


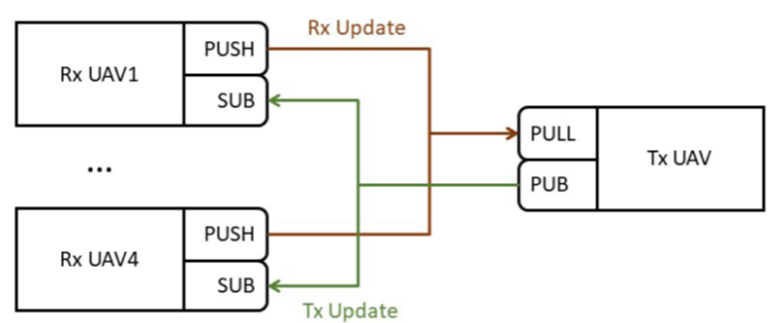

Fig. 3. The ZeroMQ messaging architecture.

chosen sockets and interconnections are shown in Fig. 3. For RX-to-TX messaging, PUSH-PULL sockets were used. Each RX has a PUSH socket for sending updates, and the TX has a single PULL socket for receiving updates from all RX UAVs. The RX UAVs can send updates whenever they are ready, without waiting for a request or an acknowledgement. For TX-to-RX communication, PUB-SUB (publisher-subscriber) sockets are used. The TX has a single PUB socket for sending updates, and each RX has a SUB socket for receiving them. Each update is received by all subscribers, so TX only needs to send a single update.

Since each RX sends an update to the TX as soon as a new range measurement is available, the TX needs to group the updates it receives, so that multilateration is only performed using ranges belonging to the same group. A simple solution is to include sequence numbers in the RX updates. Each RX has an integer counter which it increments after each range measurement, and the TX groups together updates with the same sequence numbers. However, this method has a risk that an individual RX missing a reading or using the wrong sequence number could result in errors which are hard to detect. As a simple check to prevent this, the RX updates also include timestamps, and the TX checks that the updates in each group were taken within some time interval of each other.

Finally, the UAVs should not begin sending and receiving updates until all nodes have been started. Otherwise one UAV could timeout in the time it takes to start the rest of the swarm. To ensure that the UAVs don't begin their main processing loops until all five are functional, a simple handshaking process was implemented. On start-up, each RX sends a ready message to the TX. The TX waits until it has received one from each RX, then sends a response to all of them. At this point, all UAVs begin their main loop operation.

\section{B. Target Localisation}

The localization sub-system is in charge of deriving target location from the range estimates and it operates at the TX UAV. As an input, it takes four bistatic range measurements provided by the RX UAVs together with their GPS coordinates and outputs current target location which is then communicated back to the UAVs. Generally, finding a target location from bistatic ranges requires solving a non-linear system of equations that is defined by the intersecting spheroids in $3 \mathrm{D}$, or ellipsoids in 2D. As a compromise between estimation accuracy and computational effort, for our CLS framework we

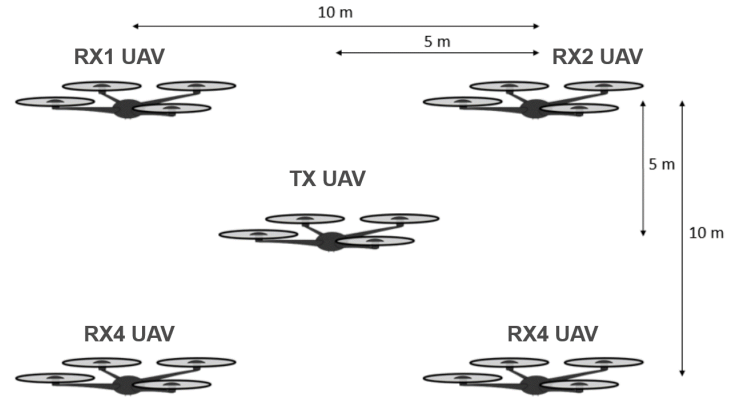

Fig. 4. UAV swarming formation with $4 \mathrm{RX}$ UAVs forming a $10 \times 10 \mathrm{~m}$ square and TX UAV in its center.

implemented a grid-based maximum likelihood (ML) location estimator. Furthermore, for testing purposes we simplified the $3 \mathrm{D}$ estimation problem into a $2 \mathrm{D}$ one by assuming that the target altitude is fixed and known, while the UAVs preserve a square formation at a fixed altitude above the target. The target lateral position is then estimated on a plane by defining an $A \times A$ search area in the target plane and splitting it into $N^{2}=(A / b)^{2}$ grid points where $b$ is the grid resolution in both dimensions. For each point on the grid, one can calculate the likelihood function $\mathcal{L}\left(\boldsymbol{p}_{\mathbf{g r}}\right)$ as the mean square distance between the estimated bistatic ranges $r_{n}$ and the candidate ranges $d_{n}$ corresponding to the grid point so that

$$
\mathcal{L}\left(\boldsymbol{p}_{\text {gr }}\right)=\sum_{n=1}^{4}\left(r_{n}-d_{n}\left(\boldsymbol{p}_{\text {gr }}\right)\right)^{2},
$$

where $\boldsymbol{p}_{\mathrm{gr}}$ indicates the grip point coordinates on the estimation plane. Since UAV positions are provided by GPS, to determine $d_{n}\left(\boldsymbol{p}_{\mathrm{gr}}\right)$ the latitude/longitude values are converted to local Cartesian coordinates and mapped onto the grid. The target location then corresponds to the point on the grid that minimizes (1).

Depending on the search area, the desired grid resolution and the update rate, calculating $\mathcal{L}\left(\boldsymbol{p}_{\text {gr }}\right)$ for each of $N^{2}$ points might become computationally expensive. To reduce computation time, we implemented a two-step approach, where we determine the most likely target locations at a coarse grid first, and then refine our estimate in the vicinity of the most probable candidate location. Note that in practice, the search area and the grid resolution will depend on the coverage area and the range resolution of the HR system.

\section{UAV Swarming}

The main goal of the swarming logic is to ensure that the UAVs are maintaining a pre-defined formation while moving along with the target. To facilitate accurate location estimation, the UAVs form a $10 \mathrm{~m} \times 10 \mathrm{~m}$ square with the TX UAV in the centre and 4 RX UAVs positioned at equal distances from it, as shown in Fig. 4. As a safety precaution, the swarming subsystem must also ensure that the UAVs do not come within $3 \mathrm{~m}$ of each another. Further, the UAVs must be prevented from moving to an unreasonable location in case of erroneous target location estimates, i.e., when the current target location 
is outside a pre-defined area, or further than a certain distance from the previous estimate, which was set to $15 \mathrm{~m}$ in our case.

The swarming rules are enforced by utilizing the centralized master-slave paradigm. The TX UAV in this case serves as a master and the RX UAVs operate as slaves. They send their GPS position updates to the TX UAV using the messaging architecture from Fig. 3. The TX UAV then performs formation checks to detect whether the formation meets the requirements. The outcome of the formation checks determines one of the three possible operation modes:

1) Move to target. This is the default operating mode which runs undisturbed unless the TX UAV requests otherwise. In this mode, UAVs derive their positions from the estimated target location distributed by the TX UAV. Each UAV is given an ID number, depending on which it applies a fixed offset to the estimated target location and moves towards that position. If the target location is deemed unreasonable, the UAVs hold their current position and wait for the next target location update.

2) Reset formation. If an average error in formation exceeds a threshold of $1 \mathrm{~m}$, the TX sends a request to reset the formation. In this mode, the GPS coordinate for the average centre of the drone formation is distributed to each UAV so that they can move to their designated positions in the formation. The mode changes back to 'move to target' if the maximum formation error doesn't exceed $0.5 \mathrm{~m}$.

3) Stop. In this mode, all drones are requested to hold their current position. This mode is included for testing purposes to terminate the operation when system failures are detected, e.g., where it may be dangerous for drones to move. It overrides any other mode and once entered it cannot be exited. UAVs need to be switched to manual control, if they reach this state.

Finally, in the current implementation the starting assumption is that all UAVs are in continuous communication. That is, the CLS framework cannot handle a UAV temporarily disconnecting then reconnecting (e.g. due to a software crash or flying out of range), or UAVs joining and leaving the swarm. Instead, if communication with any of the UAVs is lost for longer than a configurable timeout period, all UAVs will enter the stop mode and hover in place awaiting manual control. Losing communication with a UAV means that something well outside the expected system behaviour has occurred, so attempting to continue flight could result in collisions. Eventually, allowing UAVs to enter and leave the swarm may be desirable, for example to recharge UAVs on long missions, but this is outside the scope of the current implementation.

\section{EXPERIMENTAL EVALUATION}

\section{A. Target Position Server}

Since the CLS framework was developed independently of the harmonic radar sub-system, the radar range readings need to be emulated to allow testing. This was done by creating an emulated target path, and from this calculating the range readings which the radar would be expected to produce at each RX UAV position. The target path was specified as a series of GPS coordinates. The coordinates making up the path can be read either from a file, or from a GPS module connected to a laptop. To abstract away the source of the GPS coordinates from the user, a target position server (TPS) was implemented. This reads GPS coordinates from the specified source and sends them via a network socket at fixed time intervals, currently once per second. The RX receives the coordinates by connecting to the IP address and port number of this server. Each RX then uses the current target position to calculate the range which the radar would measure. Since the TX position is needed for this calculation, the TX sends its current position to the RX UAVs using the communication framework from Fig. 3. The CLS system however, operates without any prior knowledge of the target location, i.e., as if the emulated range readings were provided by the radar receivers.

\section{B. Flight Control System}

For experimental testing with actual drones, a custom control system is required that would enable flying a drone to specific GPS co-ordinates. The control system also needs to access the GPS position and other sensor information from the drone for use with the swarming and multilateration subsystems. The Aeronavics NAVIs used for the prototyping come with a Pixhawk 3DR flight controller which interfaces with the drone's sensors. The Pixhawk can also integrate with other systems so that GPS setpoints can be directly sent to it from the on-board processor. As the Pixhawk flight controller is able to handle control algorithms for flying the drone and processing the output from the sensors, the on-board Intel NUC processor is then used to handle the CLS framework. All communication between the Pixhawk and the NUC is handled via MAVROS, a communication node package for robot operating systems. Finally, to control the drones and track their positions, the ground control software QGroundControl (QGC) was used. QGC allows the user to upload the flight control software to the Pixhawk hardware as well as to switch the drones from manual control to offboard control remotely.

\section{System testing}

System validation and testing requires a simulation environment. For this purpose, we used a software-in-the-loop (SITL) approach. The Pixhawk firmware PX4 is openly available online and can be used to simulate the operation of the drone hardware. Alongside this, a physics simulator is required to provide simulated sensor information to the PX4 firmware, as well as a simulated drone to control. To this end, we used an open-source Gazebo physics simulator that allows performing SITL testing with multiple drones.

The CLS framework was first tested in the simulation environment. The target position in this case was provided by the pre-recorded target path via the TPS, while to simplify the network configuration and enable visualization in Gazebo, one UAV process was running on a separate NUC processor, and 


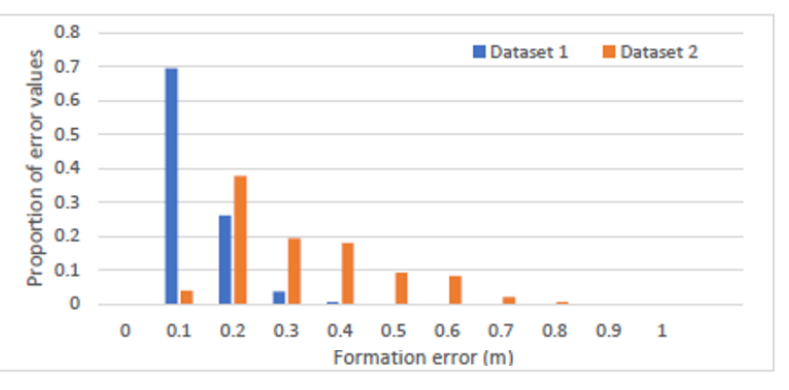

Fig. 5. Maximum formation error given two datasets of target positions: targets moving at walking speed (dataset 1) and full drone speed (dataset 2).

the other four as independent processes on the same laptop, with the laptop and NUC connected over WiFi. The update rate in this and following tests was 1 second, which means that the TPS would provide new target locations once per second and all CLS and flight control operations need to be completed within this time. Using the developed workflow, it was possible to simulate all five drones flying in a formation based off GPS co-ordinates delivered by the CLS, while monitoring their progress using QGC. In the absence of range errors, the target location estimation accuracy was determined by the grid resolution which was set to $b=1 \mathrm{~m}$. Fig. 5 shows the maximum formation error, defined as the maximum difference between drone's position and its predefined place in the formation, for targets moving at walking speed (dataset 1), and full drone speed (dataset 2).

In field trials, a similar workflow was used with the target path now being emulated by a person carrying a laptop with a GPS module providing its co-ordinates via the TPS. Also, a separate laptop was set up with an external radio link to the flying drones running QGC for drone monitoring and debugging. A manual radio controller was connected to the flying drones using the same radio link and a pilot was on standby to take control of the drones if necessary. The drone altitude was fixed at $20 \mathrm{~m}$, while the target altitude corresponded to about $1 \mathrm{~m}$. The drones were launched manually and once in the air switched to offboard mode using QGC. The tests were performed on Ilam Fields in Chirstchurch, New Zealand with first one and then two drones in the air, and the rest simulated in Gazebo. Figure 6 illustrates the test setup. Both tests went ahead successfully with the swarm of drones being able to follow the target movement within the $1 \mathrm{~m}$ accuracy requirement.

\section{Conclusions}

In this contribution, we present the results of the design, implementation and testing of a proof-of-concept prototype of a distributed system for tracking of moving targets with an autonomous swarm of UAVs. In a simplified setup with up to two UAVs in the air and the rest emulated in a flight simulator, a swarm of UAVs was shown to be able to locate and follow a moving target in a pre-defined formation. Further development and testing is required to insure that the designed system is capable of tracking faster-moving targets in 3D.

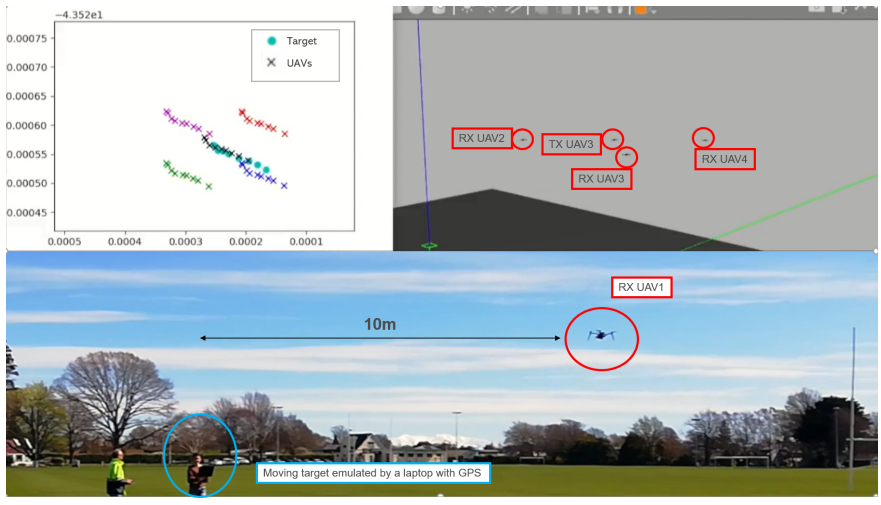

Fig. 6. Flight test with one real-life UAV (bottom picture) flying in formation with four simulated UAVs (top right picture). Top left picture illustrates the UAV positions (colored X's) with respect to the target position (teal dots).

\section{REFERENCES}

[1] C. A Hallmann, M. Sorg, E. Jongejans, H. Siepel, N. Hofland, H. Schwan, W. Stenmans, A. Müller, H. Sumser, T. Hörren, et al., "More than 75 percent decline over 27 years in total flying insect biomass in protected areas," PloS one, vol. 12, no. 10, pp. e0185809, 2017.

[2] D. Mascanzoni and H. Wallin, "The harmonic radar: a new method of tracing insects in the field," Ecological entomology, vol. 11, no. 4, pp. 387-390, 1986.

[3] M. E O'Neal, D. A Landis, E. Rothwell, L. Kempel, and D. Reinhard, "Tracking insects with harmonic radar: a case study," American Entomologist, vol. 50, no. 4, pp. 212-218, 2004.

[4] B. G Colpitts and G. Boiteau, "Harmonic radar transceiver design: miniature tags for insect tracking," IEEE Transactions on Antennas and Propagation, vol. 52, no. 11, pp. 2825-2832, 2004.

[5] S. Bottigliero, D. Milanesio, M. Saccani, R. Maggiora, and M. M. Viscardi, A.and Gallesi, "An innovative harmonic radar prototype for miniaturized lightweight passive tags tracking," in 2019 IEEE Radar Conference (RadarConf). IEEE, 2019, pp. 1-6.

[6] A. Lavrenko, B. Litchfield, G. Woodward, and S. Pawson, "Design and evaluation of a compact harmonic transponder for insect tracking," IEEE Microwave and Wireless Components Letters, vol. 30, no. 4, pp. 445-448, 2020.

[7] G. Storz and A. Lavrenko, "Compact low-cost FMCW harmonic radar for short range insect tracking," in 2020 IEEE International Radar Conference (RADAR), 2020, pp. 642-647.

[8] N. Tahir and G. Brooker, "Efficient design of harmonic transponder for UAV based harmonic tracker," in 5th International Conference on Intelligent Sensors, Sensor Networks and Information Processing (ISSNIP 09), 2009.

[9] Q. S. Le, J. Kim, J. Kim, and H. Il Son, "Report on work in progress of small insect tracking system using autonomous uav," in 2017 14th International Conference on Ubiquitous Robots and Ambient Intelligence (URAI). IEEE, 2017, pp. 242-243.

[10] S. Aljaser, "Miniaturization of a low power harmonic radar for UAV use," 2019.

[11] D. Psychoudakis, W. Moulder, H.g Chen, C.-C.and Zhu, and J. L Volakis, "A portable low-power harmonic radar system and conformal tag for insect tracking," IEEE Antennas and Wireless Propagation Letters, vol. 7, pp. 444-447, 2008.

[12] N. Tahir and G. Brooker, "Recent developments and recommendations for improving harmonic radar tracking systems," in Proceedings of the 5th European Conference on Antennas and Propagation (EUCAP). IEEE, 2011, pp. 1531-1535.

[13] M.-L. Hsu, S.-J. Jan, Z.-M. Tsai, H. Wang, F.-R. Chang, P.-H. Jau, K.-Y. Lin, and E.-C. Yang, "Portable 9.4/18.8 GHz harmonic radar system using pulse pseudorandom code principle," in 2015 European Microwave Conference (EuMC). IEEE, 2015, pp. 885-888.

[14] G. J Mazzaro, K. A Gallagher, A. F Martone, K. D Sherbondy, and R. M Narayanan, "Short-range harmonic radar: Chirp waveform, electronic targets," in Radar Sensor Technology XIX; and Active and Passive Signatures VI. International Society for Optics and Photonics, 2015, vol. 9461, p. 946108. 